\title{
The Influence of Parametric Design Tools on Increasing Creativity in the Furniture Design Process
}

\author{
Shalaw Hamza Hamad ${ }^{1} \&$ Husein Ali Husein ${ }^{2}$ \\ ${ }^{1}$ Department of Architecture, Faculty of Engineering, Tishk International University, Erbil, Iraq \\ 2 Department of Architecture, Faculty of Engineering, Salahaddin University, Erbil, Iraq \\ Correspondence: Shalaw Hamza Hamad, Tishk International University, Erbil, Iraq. \\ Email: shallaw.hamza@tiu.edu.iq
}

Doi: 10.23918/eajse.v6i1p199

\begin{abstract}
This paper tries to investigate the influence of parametric design tools on increasing creativity of furniture designers, hence the most difficult issue for a designer is the creative thinking and bringing new ideas into life. The methodology of this paper is to make a protocol analysis to find criteria of creativity based on the reviewed literature, then create a measurement scale based on these criteria to evaluate the creative aspects of parametric design furniture's, and a comparison study has been made between parametric design furniture designed with geometrical model-based design. A survey prepared and distributed among 30 architects who had experience in furniture design to assess the creativity level of each furniture which contained three categories (chairs, shelves, and built-in furniture). The participants were 22 males and 8 females. From analyzing results, we can find that $65.38 \%$ of the participants said they have used parametric design tools in the average of ( 3 years' experience) while the other $34.62 \%$ said they didn't use it before. $\mathbf{9 2 . 3 1 \%}$ from the respondents think that parametric design increases creativity, and the rest disagrees. The results of the first part show that participants rated the selected parametric furniture based on criteria of creativity as following: For Originality 4/5, aesthetic 4/5, Function 3.5/5, elegancy $4 / 5$, and Innovation $4 / 5$. While the second part of the survey which a comparison; the median for parametric furniture's creativity level was 4 while the median for geometric based modelled was 3 . From the results, we can conclude that parametric design tools indeed help designers to do creative designs more than geometric based design tools. For future studies, we suggest investigating the role of parametric design in the interior design process in total, hence we only studied its influence on the furniture design process.
\end{abstract}

Keywords: Parametric Design, Creativity, Design Process

\section{Introduction}

Though nowadays parametric design is mainly linked to the technological world, it has existed much longer before in tie of appearing (CAD). While advances in technology have made parametric tools more efficient but the idea and its origin were as early as limiting the human proportions and mathematical rules in architecture as in the Greek and Roman architecture for instance, which can be considered as a parametric. Parametric design is usually described as a reckoning design concept generation which provides you with many options while making decisions for a design (Blosiu, 1999). It can be considered as a new style in architecture and which day by day increases its contribution to increasing creativity of designers (Schumacher, 2009).

Improving compactivity has always been one of the industrial goals (Antonovsky, 1996), which encourages companies to find solutions by using Computer-aided design (CAD). After developing computer software from 2D to 3D modelling, new techniques and tools appeared such as parametric design and modelling. Cost efficiency and structural analysis for furniture have been achieved by using CAD software-Parametric design by Barros et al. (Oxman, 2008). In 2008 mathematical equations 
have been used by Pan and Wang in the furniture design process. Time reduction and flexibility in creating different versions are some of the main benefits of using parametric modelling (Qian, 2007).

Mainly we can find parametric design in the CAD software applications as a shape of tools, that the 3D models edited and by changing the parameters the unexpected results will be found, although it has other utilization in ceramic industry (Abdelmohsen, 2009) or construction elements (Chien, 2012). In both cases, the starting point was a parameterized three-dimensional model. Using design tables in the furniture industry to implement parametric design is useful when a large order is needed to be fulfilled with different sizes of the same model of furniture, as it reduces work time and the possibility of errors.

\subsection{Machine Human Relationship}

The parametric design process is mainly depending on the relationship between human and machines. As it's suggested by Deleuze and Guattari machines life exist, and they and their lives are linked with human's life strongly. Parametric modelling and design tools considering this connection enable the designers to generate models that are beyond our imagination. Defiantly these tools had given architects flexibility in choosing multiple versions and generate creative ideas in the conceptual design stage. Figure 1 shows that the relation between humans and computers is very strong. We code our computer and this computer will lead another one to achieve a higher level.

\subsection{Computers and Concept}

Using computer-aided programs architects' and furniture designers' creativity was increased and they made innovative designs that cannot be drawn by hand easily and complicated problems were solved. Making the design a piece of art and special is the job of designers. While the designer should play a significant role in enhancing the cultural, social, environmental, and economic aspects of the site, computers play their role in increasing the aesthetic and creativity of that design. The range of creativity outcomes designed by these programs depend on the ability to use it. Integrating these with parametric tools will increase the flexibility of designers to choose the best model among unlimited opportunities of the design to make the right decision enhancing health and welling of the users.

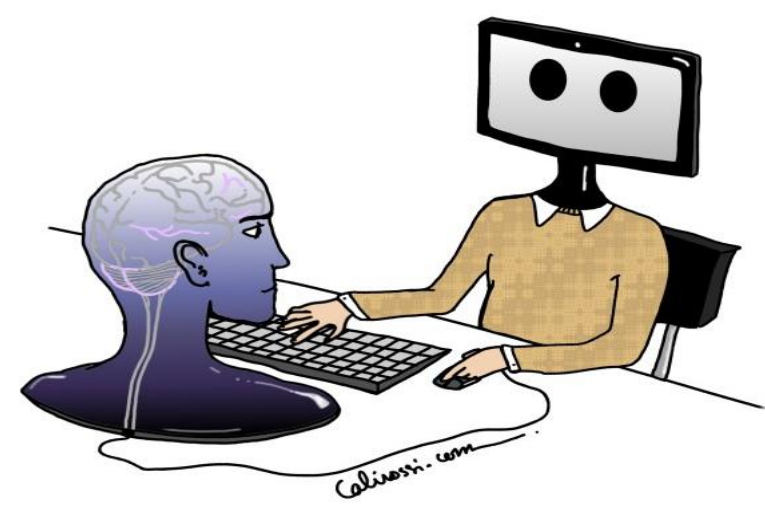

Figure 1: shows the dependence of human and computer on each other (www.wekmedia.com)

\subsection{Parametricism}

Patrik Schumacher is considered as the father of parametric design; he entitles innovative methods and tools as parametricism (Zarei, 2012). This new method and theory can be applied to all scales starting 
from a single item to urban scale in design and utilization process. The parametric design itself is a new approach and style and as Schumacher said it's not just about style but it can change how people think about buildings and it will affect people's perception (Schumacher, 2012). Figure 2 shows how this parametric tool been used by Zaha Hadid and Patrick Schumacher with the result of ultimate level of creativity.

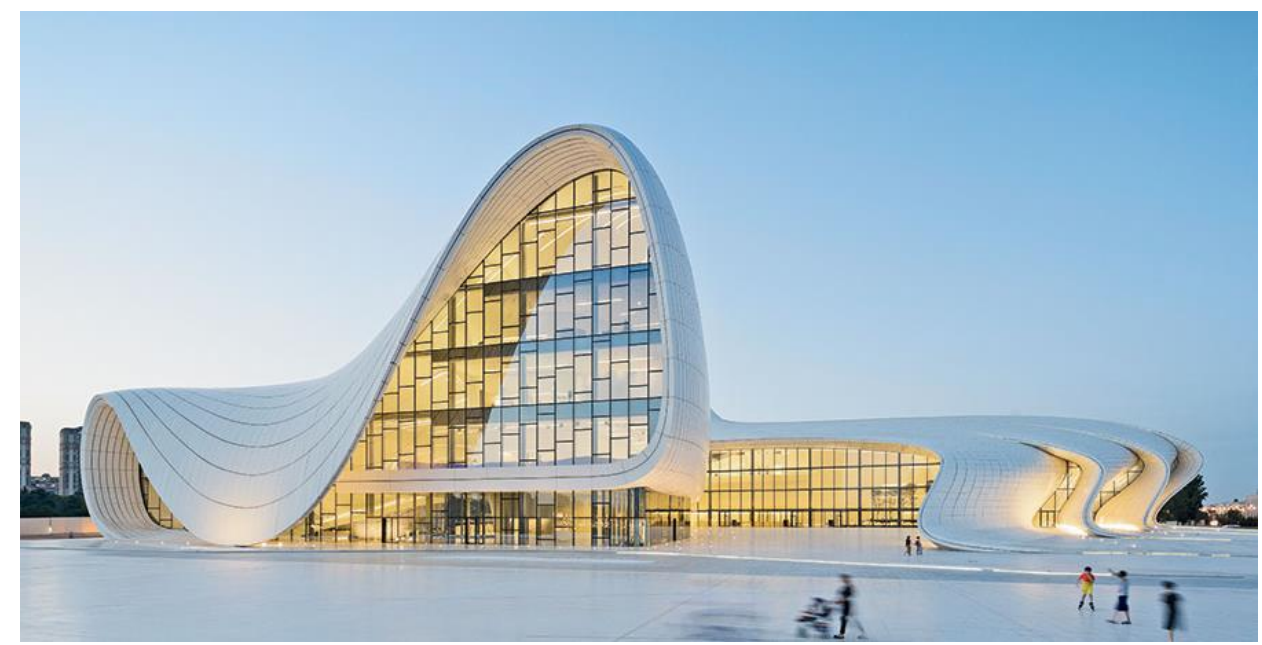

Figure 2: Heydar Aliyev culture center by Zaha Hadid used parametric design

\subsection{Problem Statement}

In the design concept process, usually the most important thing is to think creatively and generate new ideas; that most of the designers find difficulties in generating something innovative, creative and make their ideas actualized. It is where the parametric tools will help to generate infinite options from one single element.

\subsection{Hypothesis of the Research}

Parametric tools help designers to generate new ideas and produce creative designs and reduce consumed time through the conceptual design process of furniture design.

\section{Literature Review}

From the last years of the twentieth century, that $3 \mathrm{~d}$ modelling tools appeared and architects began to replace 2D modelling programs with 3d modelling programs (Bilda, 2003). We can realize the same revolution with BIM nowadays which is challenging all other types of computer modelling in design, architectural and engineering industry. Rhino Grasshopper, ArchiCAD and Graphic soft para 3d plugin in 3Ds max, Google SketchUp and many others are software that provides parametric modelling and they help designers to create something new and creative. The philosophy of parametric design tools is quite simple, and it depends on the variation of its parameters that you can choose between an infinite number of options instead of limited options in the design concept process (Woodbury, 2010). By using these methods, very complex problems and project management can be solved and the final design look depends on the various values of each parameter, though there should be at least one constant between parameters (Kolarevic, 2003). Have a look at Figure 3 for a better understanding. 


\section{A Single Column.}

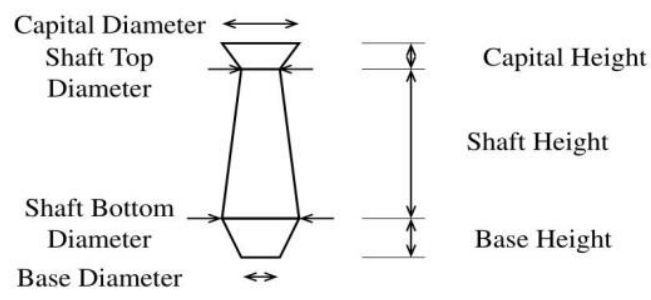

- Column has 7 dimensions

Figure 3: Explaining how parametric design method works

According to Kolarevic; those parameters are usually factoring in Architectural design, surrounding environment or inner enclosed environment that can affect the design process. Although parametric design method is mainly used for form-finding for architectural design, nowadays it is in use in structural and problem-solving complex projects. The use of parametric modelling has increased by the rise of the sustainability theory. Nowadays Zaha Hadid architects, Gehry and partners, Norman Foster and partners, BIG architects, many others are using this method in their design that leads them to become world leader-architects. Sometimes special software created for their needs but usually, they depend on a grasshopper in their design (Lee, 2015)

Combination of Rhino and its plugin grasshopper can lead designers to create innovative design form and best diction making and generate new ideas. In case we can find that followers of geometric modelling approach could find creative new ideas in their design but, the consumed time is much more than parametric design method and also the options are very limited (Iordanova, 2009). New Ideas and novelty forms can be easily generated as Schnabel declared in his study that parametric design can produce unpredicted results that it was impossible to think about without the help of the parametric design tools as in figure 4. A protocol study has been suggested to compare parametric design tools with common tools for geometric design tools to understand the creativity of parametric design tools and the shift that can be achieved in conceptualization by using this approach. However, designer behaviours cannot be neglected and may have effects on the results.

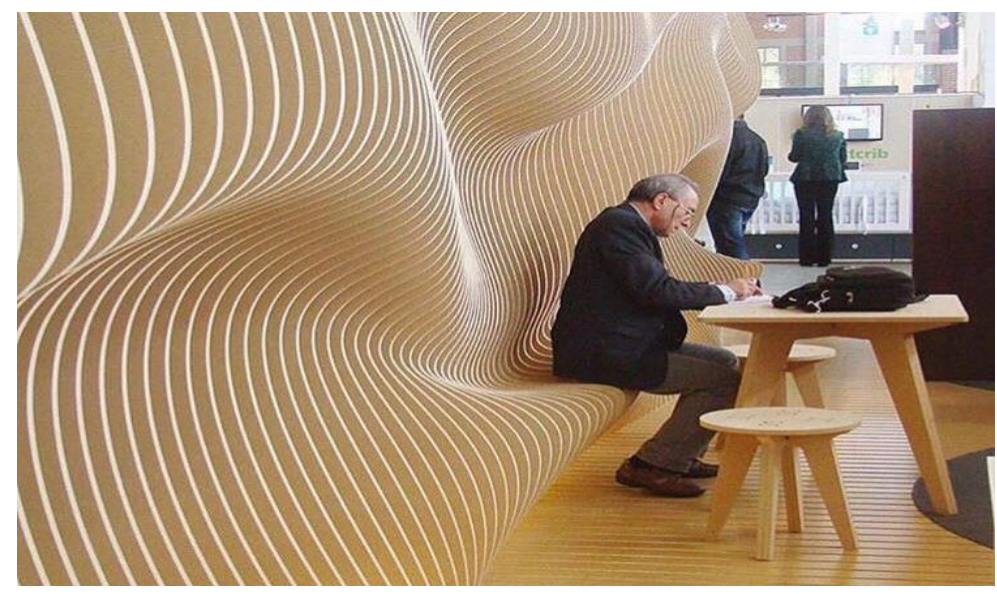

Figure 4: A parametric wall that can be used as a chair in a restaurant (www.archdaily.com) 
In this study protocol analysis will be done to highlight this gap. As it's suggested by Lee et al. in their study to use this method to evaluate the creativity of parametric designs, we can find the same approach in Chien and Yeh who found unexpected results while they were investigating parametric design and its outcomes (Chien, 2012). Nevertheless, there isn't a better way than this comparison to understand and clarify the differences between parametric design and traditional design tools in its application in the field of design and engineering in general. For instance, to evaluate the creativity level of parametric design we should understand how to measure creativity.

As it is suggested by Cropley, Kaufman and Cropley (2011), creativity can be measured by these indicators:

- Innovation

- Aesthetics

- Function

- Elegance

- Original

We can use this checklist to evaluate and compare furniture designed with parametric design methods $\&$ traditional modelling tools based on the protocol analysis method.

\section{Methodology}

This study applies protocol analysis to compare parametric design and geometric-based furniture design. A deep analysis has been done through available literature to find measurements of creativity then a survey questionnaire was prepared based on those measurement criteria to evaluate a global parametric furniture in the first part. In the second part a comparison has been made between 6 different types of furniture from three categories (Chair, Built-in, and shelves) in both styles. The data collected from the survey is a statistical analysis by Zoho survey analysis tools. The survey distributed between 30 designers who have experience in furniture design to evaluate the level of creativity of parametric furniture designs in comparison to geometric modelling features.

These are furniture used in a comparison part of the survey:

1.Parametric builtin furniture

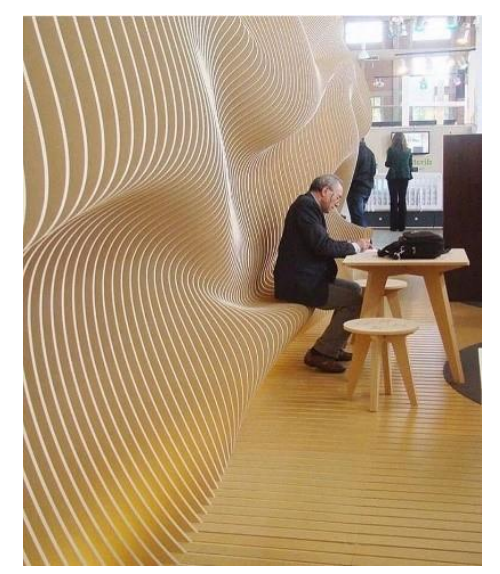

2.Parametric Chair
Geometric modelling builtin furniture

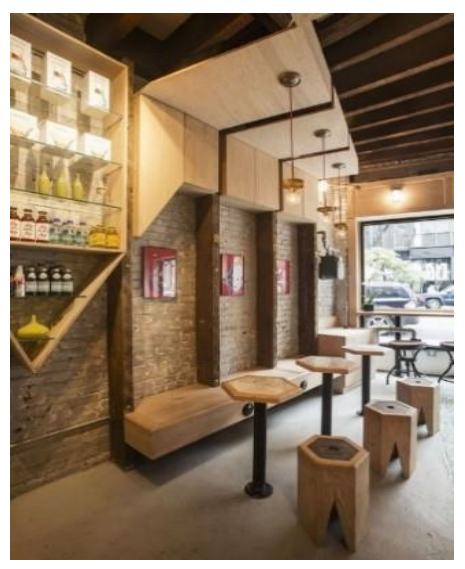

Geometric Based Chair 


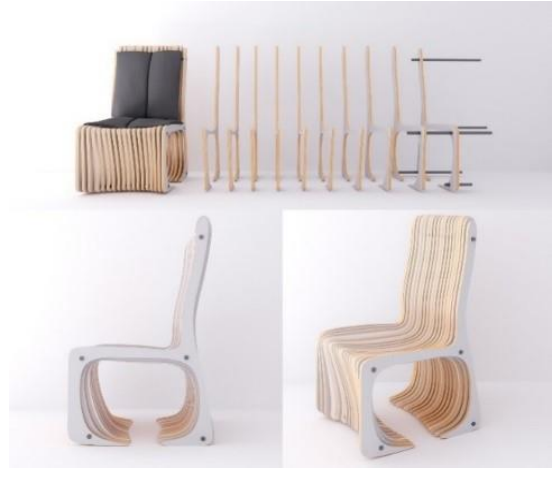

3.Parametric Shelve design

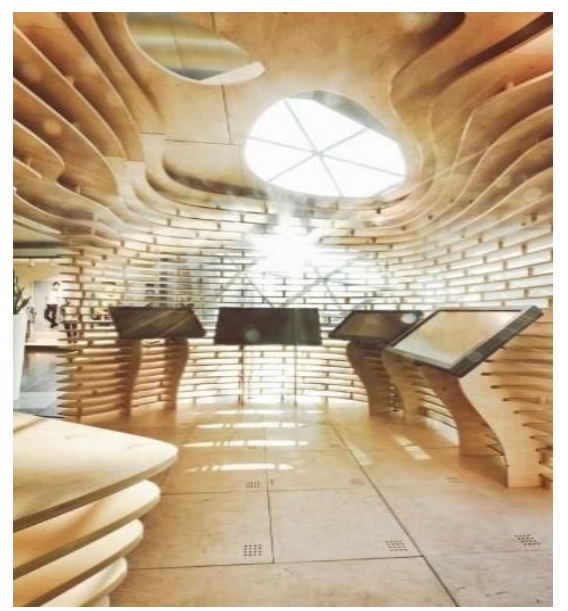

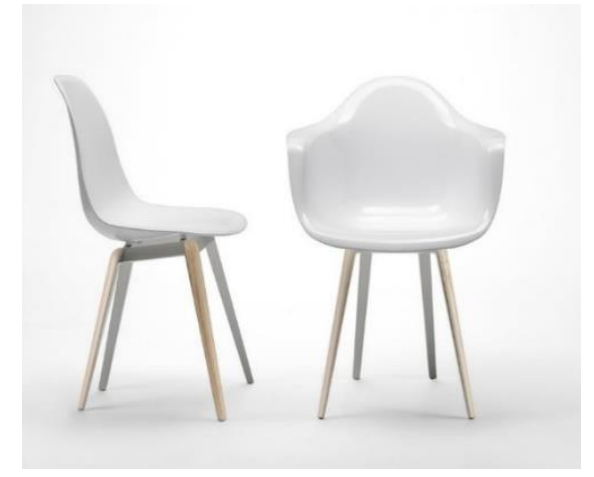

Geometric based modeling Shelve design

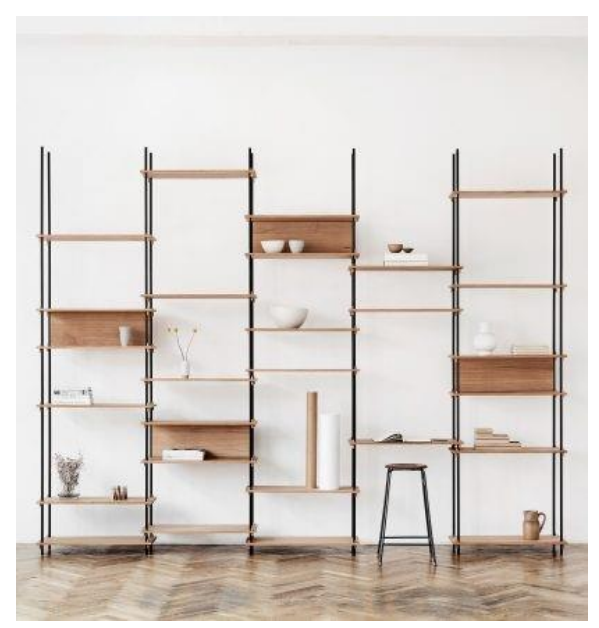

\section{Results and Findings}

The participants were 22 males and 8 females. $65.38 \%$ of the participants said they have used parametric design tools in the median of (3 years experience) while the other $34.62 \%$ said they didn't use it before. $92.31 \%$ from the respondents think that parametric design increases creativity, and the other part does no think it does.

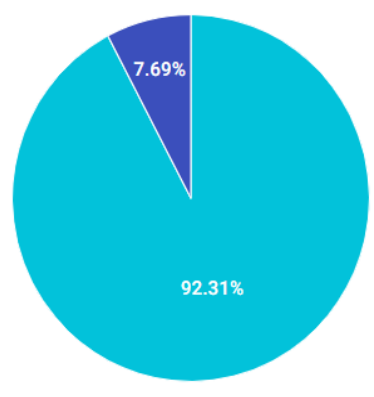

Figure 5: A pie chart which shows the answers for whether the participants think parametric design tools increase creativity level 
The participants in this survey had information about parametric design by $19.23 \%$ very good, 38.46 $\%$ good, $34.62 \%$ neutral, and $7.69 \%$ said their information on design parametric design was poor. $65.38 \%$ said they used parametric design tools before and the median of their experiences was 3 years, while $34.62 \%$ said they didn't use it before.

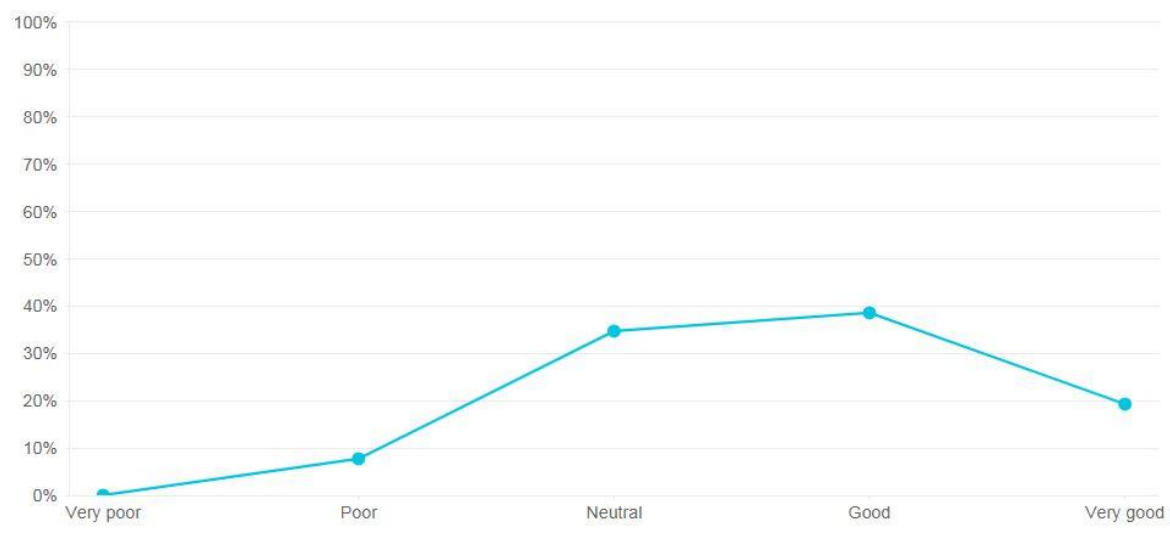

Figure 6: A line chart explains the amount of information that participants have on parametric design

The participants evaluated this parametric furniture according to creativity measurements (Innovative, Aesthetic, Function, Elegance, Original).

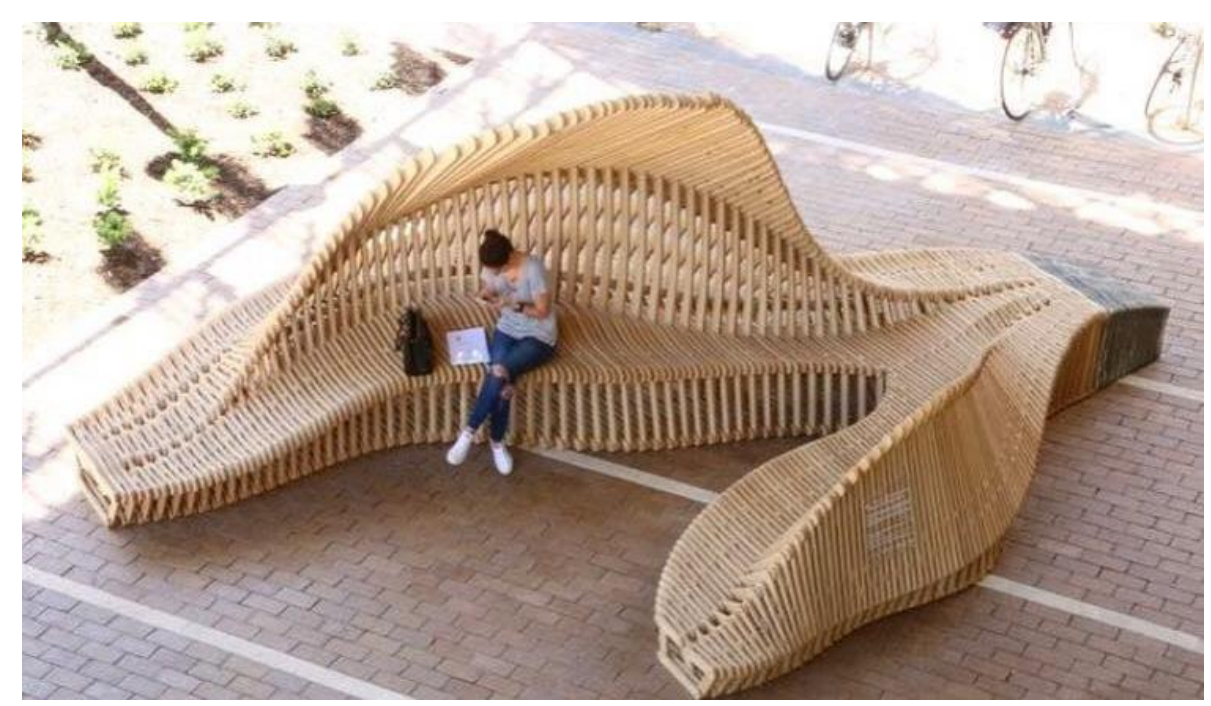

Figure 7: The multifunctional parametric used in the survey to be evaluated by the measurement tool that we created in a theoretical framework in this study (www.archdaily.com)

And the results were as follows: from originality scale, they said: $54.17 \%$ it is original. $37.5 \%$ normal, $8.33 \%$ aboriginal. From elegance scale they said: $16.67 \%$ it's very elegant. $50 \%$ elegant, $25 \%$ normal, $8.33 \%$ inelegant. In terms of function responses: $8.33 \%$ it's very functional, $41.67 \%$ functional, $37.50 \%$ normal, $8.33 \%$ not functional and $4.17 \%$ were not sure. In aesthetic scale, the results were: $16.67 \%$ very beautiful, $45.83 \%$ beautiful, $20.83 \%$ neutral, and $16.67 \%$ not beautiful. In the innovation scale, the responses were: $23.06 \%$ very innovative, $53.85 \%$ innovative, $11.54 \%$ neutral, and $11.54 \%$ absolutely not innovative. 
Table1: The percentage ratings scale

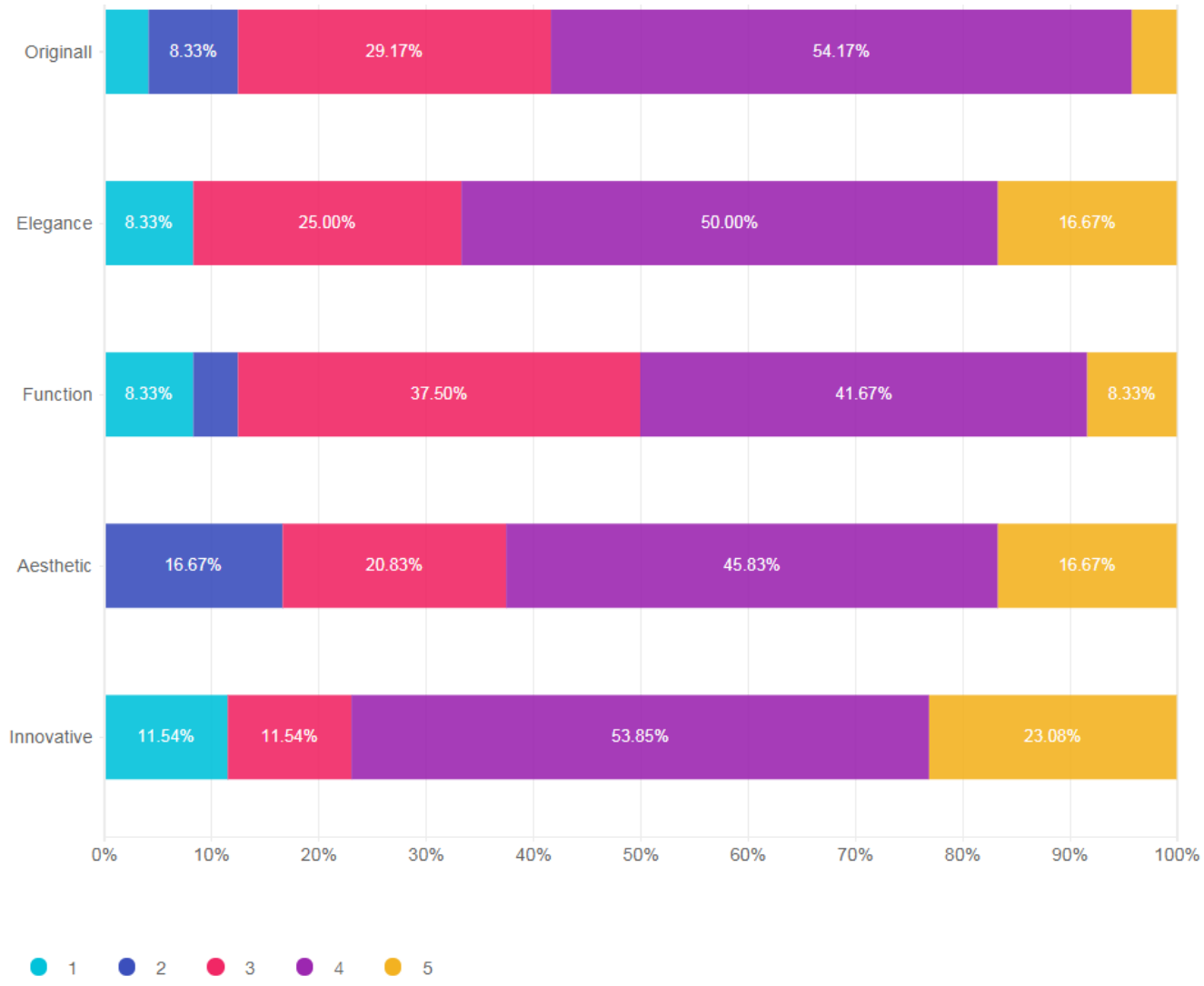

Statistic analysis has been done for collecting data from the participant responses: for the first option originality scale: mean of the answers was 3.77 , median $=4$, std. deviation $=1.18$, variance $=1.38$. For elegance scale answers: mean $=3.62$, median $=4$, std. deviation $=0.97$ variance $=0.94$. In a matter of function of the furniture, the statistics were: mean $=3.338$, median 3.5 , std. deviation $=1.01$, variance 1.03. For elegance scale the statistics were: mean $=3.67$, median $=4$, std. deviation $=1.05$, variance 1.1 . From the originality scale: mean $=3.46$, median 4 , std. deviation $=0.8$, variance 0.78 .

Table 2: Statistical analysis of parametric furniture evaluation by designers

\begin{tabular}{|c|c|c|c|c|c|c|c|c|c|c|}
\hline \multirow{2}{*}{$\begin{array}{l}3 \\
0 \\
\alpha\end{array}$} & \multicolumn{2}{|l|}{$\begin{array}{l}\mathbb{8} \\
\approx \\
\approx \\
\approx\end{array}$} & \multicolumn{2}{|c|}{ 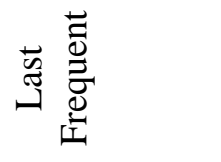 } & \multicolumn{2}{|c|}{ 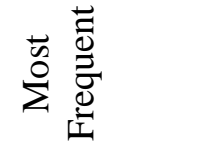 } & \multirow{2}{*}{ 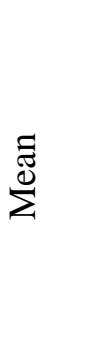 } & \multirow{2}{*}{ 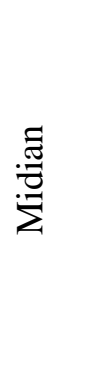 } & \multirow{2}{*}{ 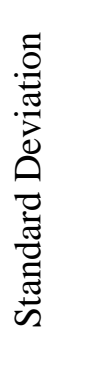 } & \multirow{2}{*}{ 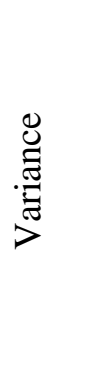 } \\
\hline & ह్ & $\stackrel{0}{\varphi}$ & 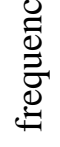 & $\frac{\stackrel{0}{J}}{\sqrt[\pi]{>}}$ & 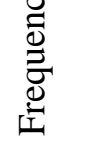 & 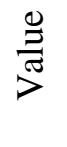 & & & & \\
\hline Innovation & 1 & 5 & 3 & 1,3 & 14 & 4 & 3.77 & 4 & 1.18 & 1.38 \\
\hline Aeshetic & 2 & 5 & 4 & 2,5 & 11 & 4 & 3.62 & 4 & 0.97 & 0.94 \\
\hline Function & 1 & 5 & 1 & 2 & 10 & 4 & 3.38 & 3.5 & 1.01 & 1.03 \\
\hline Elegance & 1 & 5 & 2 & 1 & 12 & 4 & 3.67 & 4 & 1.05 & 1.1 \\
\hline Originall & 1 & 5 & 1 & 1,5 & 13 & 4 & 3.46 & 4 & 0.88 & 0.78 \\
\hline
\end{tabular}


The interpretation of the second part of the survey was a comparison between furniture and parametric design approach vs furniture and the first comparison was between built-in-furniture. The results were as follows: for the parametric wall $30.00 \%$ rated it as very creative, $40.00 \%$ creative, $15.00 \%$ neutral, $5.00 \%$ uncreative, and $10.00 \%$ strongly uncreative. And the median of all answers was 4 .

Table 3: A pie chart and a statisctical table analysis for answers of the survey about parametric designed furniture

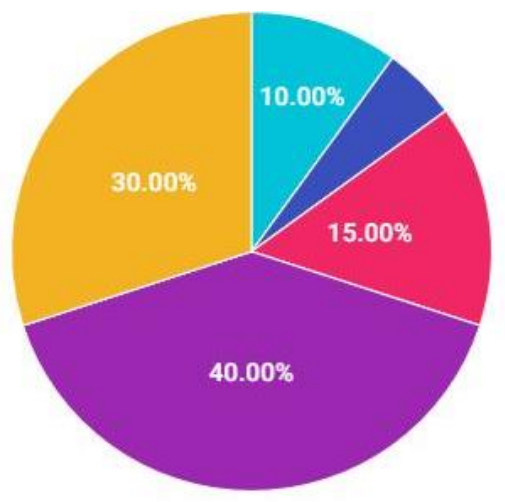

\begin{tabular}{|c|c|c|c|c|c|c|c|c|c|}
\hline \multicolumn{2}{|c|}{ Range } & \multicolumn{2}{c|}{ Last Frequent } & \multicolumn{2}{c|}{ Most Frequent } & Mean & Midian & $\begin{array}{c}\text { Standard } \\
\text { Deviation }\end{array}$ & Variance \\
\cline { 1 - 5 } From & To & frequency & Value & Frequency & Value & & & & \\
\hline 1 & 5 & 1 & 1 & 8 & 4 & 3.75 & 4 & 1.25 & 1.57 \\
\hline
\end{tabular}

Ratings for geometric based model furniture was as follows:13.64 \% very creative, $63.64 \%$ neutral, $9.09 \%$ uncreative, $9.09 \%$ strongly uncreative, and the median of all answers was 3 which means that designers rated it as normal.

Table 4: A pie chart and a statisctical table analysis for answers of the survey about geometric model based furniture

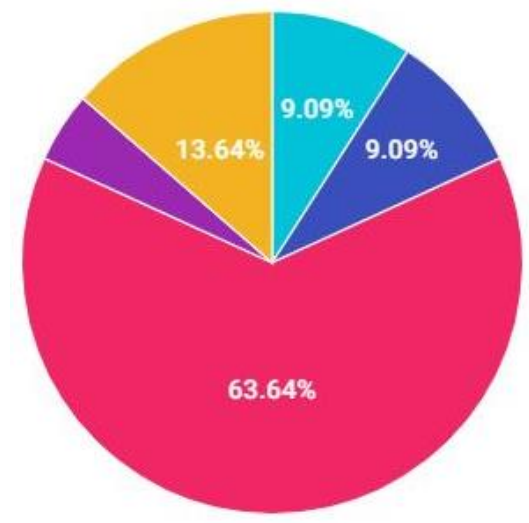

\begin{tabular}{|c|c|c|c|c|c|c|c|c|c|}
\hline \multicolumn{2}{|c|}{ Range } & \multicolumn{2}{c|}{ Last Frequent } & \multicolumn{2}{c|}{ Most Frequent } & Mean & Midian & $\begin{array}{c}\text { Standard } \\
\text { Deviation }\end{array}$ & Variance \\
\cline { 1 - 5 } From & To & frequency & Value & Frequency & Value & & & & 1.09 \\
\hline 1 & 5 & 1 & 4 & 14 & 3 & 3.05 & 3 & 1.05 & 1.05 \\
\hline
\end{tabular}


The second comparison was between shelve designs and the results were as follows: $27.27 \%$ very creative, $40.91 \%$ creative, $22.73 \%$ normal, and $9.09 \%$ very uncreative and the median of all answers was 4 (creative).

Table 5: A combination of the pie chart and a statistical table analysis for answers of the survey about parametric shelves

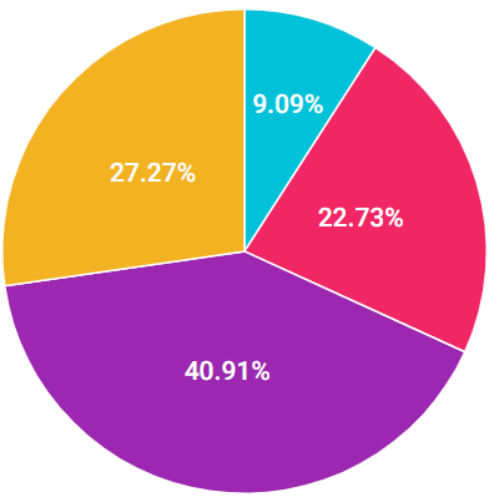

\begin{tabular}{|c|c|c|c|c|c|c|c|c|c|}
\hline \multicolumn{2}{|c|}{ Range } & \multicolumn{2}{c|}{ Last Frequent } & \multicolumn{2}{c|}{ Most Frequent } & Mean & Midian & $\begin{array}{c}\text { Standard } \\
\text { Deviation }\end{array}$ & Variance \\
\cline { 1 - 5 } From & To & frequency & Value & Frequency & Value & & & & \\
\hline 1 & 5 & 2 & 1 & 9 & 4 & 3.77 & 4 & 1.15 & 1.33 \\
\hline
\end{tabular}

The interpretation to geometrical based modelling design shelves ratings from the survey is as follows: $22.73 \%$ very creative, $13.67 \%$ creative, $31.82 \%$ normal, $18.18 \%$ uncreative, $13.64 \%$ very uncreative. The median of the rating was 3 (normal).

Table 6: A combination of the pie chart and a statistical table analysis for answers of the survey about parametric shelves

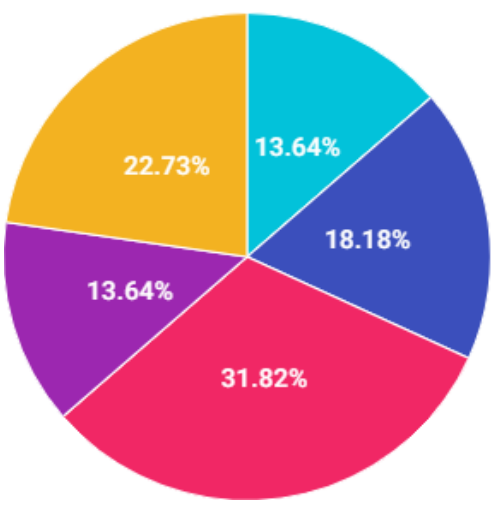

\begin{tabular}{|c|c|c|c|c|c|c|c|c|c|}
\hline \multicolumn{2}{|c|}{ Range } & \multicolumn{2}{c|}{ Last Frequent } & \multicolumn{2}{c|}{ Most Frequent } & Mean & Midian & $\begin{array}{c}\text { Standard } \\
\text { Deviation }\end{array}$ & Variance \\
\cline { 1 - 7 } From & To & frequency & Value & Frequency & Value & & & & \\
\hline 1 & 5 & 3 & 1,4 & 7 & 3 & 3.14 & 3 & 1.36 & 1.84 \\
\hline
\end{tabular}

The third comparison was between parametric chart and a geometric modelling based chair. The interpretation of the results is as follows: for the parametric chair, the statistical analysis was $31.82 \%$ 
very creative, $36.36 \%$ creative, $18.18 \%$ normal, $4.55 \%$ uncreative, and $9.09 \%$ of the responses said it's very uncreative. The median of all answers was 4 which means ( creative).

Table 7: A pie chart and a statisctical table analysis for answers of the survey about parametric chair

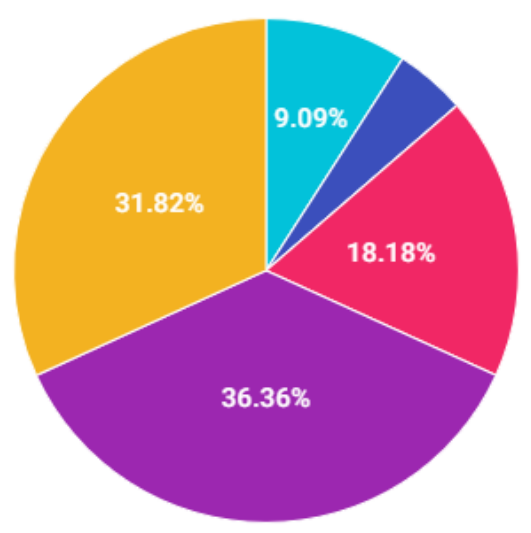

\begin{tabular}{|c|c|c|c|c|c|c|c|c|c|}
\hline \multicolumn{2}{|c|}{ Range } & \multicolumn{2}{c|}{ Last Frequent } & \multicolumn{2}{c|}{ Most Frequent } & Mean & Midian & $\begin{array}{c}\text { Standard } \\
\text { Deviation }\end{array}$ & Variance \\
\cline { 1 - 7 } From & To & frequency & Value & Frequency & Value & & & & \\
\hline 1 & 5 & 1 & 2 & 8 & 4 & 3.77 & 4 & 1.23 & 1.52 \\
\hline
\end{tabular}

While the results for a geometric modelled chair was somehow below the parametric chair. The rating was like that: $19.05 \%$ said it's very creative, $14.29 \%$ creative, $38.10 \%$ normal, $19.05 \%$ uncreative, and $9.52 \%$ said it's very uncreative. And the median the rating was 3 which means normal.

Table 4: A pie chart and a statisctical table analysis for answers of the survey about geometric model baseed chair

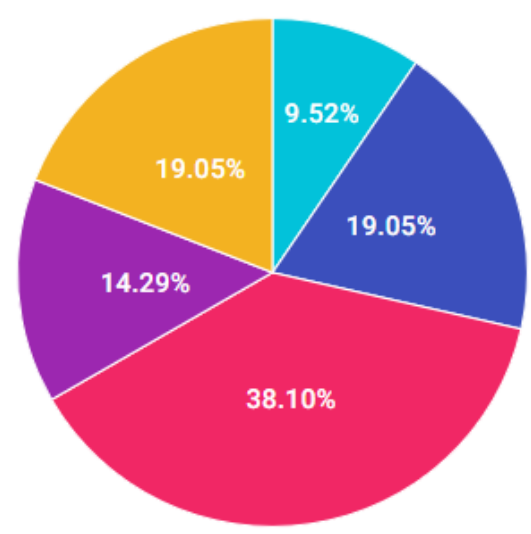

\begin{tabular}{|c|c|c|c|c|c|c|c|c|c|}
\hline \multicolumn{2}{|c|}{ Range } & \multicolumn{2}{c|}{ Last Frequent } & \multicolumn{2}{c|}{ Most Frequent } & Mean & Midian & $\begin{array}{c}\text { Standard } \\
\text { Deviation }\end{array}$ & Variance \\
\cline { 1 - 7 } From & To & frequency & Value & Frequency & Value & & & & \\
\hline 1 & 5 & 2 & 1 & 8 & 3 & 3.14 & 3 & 1.24 & 1.53 \\
\hline
\end{tabular}




\section{Limitations of the Study}

This paper is concentrating on parametric design tools and their effects on increasing the creativity level of furniture designers in the conceptual stage furniture design process.

\section{Conclusion}

Parametric design tools are mainly known as an arithmetic design concept generation which provides more options than the human mind can cover it while making decisions in the conceptual design process. Hence generating new ideas and creativity are the issues that most furniture designers find difficulties with because it requires imagination and a high level of creativity. The methodology of this paper was to make a protocol analysis, find criteria of creativity to be able to create a measurement scale to evaluate the creativity of parametric design furniture, and in the next step a comparison study was made between furniture that was designed by parametric design tools vs pieces of furniture that was designed with geometrical model-based design. A survey was prepared and distributed to 30 architects who had experience in furniture design to rate the creativity level of each furniture which contained three categories (chair, shelves, and built-in furniture). The participants were 22 males and 8 females. $65.38 \%$ of the participants said they used parametric design tools in the median of (3 years' experience) while the other $34.62 \%$ said they didn't use it before. $92.31 \%$ of the respondents think that parametric design increases creativity, and the other part responded that it did not help. From the analysis results of the first part we can see that participants rated the selected parametric furniture as follows: In originality $4 / 5$, aesthetic $4 / 5$, function $3.5 / 5$, elegancy $4 / 5$, and innovation $4 / 5$. While In the second part of the survey which architects asked to set creativity level of furniture, the median for parametric furniture creativity level was 4 while the median for geometric based modelled was 3 . From the results, we can conclude that parametric design tools indeed helps designers to do creative designs more than geometric based design tools. For future studies we suggest to investigate the role of parametric design in the interior design process in total, hence we only studied its influence on the furniture design process.

\section{Future Suggestions}

Since this paper only analyzed the effects on the parametric design on the creativity of the designers; the researchers, therefore, it is recommended to concentrate on kinetic interior design structure, wall and materials used in inner spaces and its effect on the psychology and behaviours of the users.

\section{References}

Abdelmohsen, S. M., \& Do, E. Y. L. (2009). Analysing the significance of problem solving expertise and computational tool proficiency in design ideation.

Antonovsky, A. (1996). The salutogenic model as a theory to guide health promotion. Health

Promotion International, 11(1), 11-18.

Architectural Design, 79, 14-23.

Bilda, Z., \& Demirkan, H. (2003). An insight on designers' sketching activities in traditional versus digital media. Design Studies, 24(1), 27-50.

Blosiu, J. O. (1999). Use of synoptics as an idea seeding technique to enhance design creativity. Paper presented at the 1999 IEEE international conference on the Systems, Man, andCybernetics, 1999 (IEEE SMC '99), Tokyo (Vol. 3, pp. 1001-1006). IEEE.

Branko, K. (2003). Architecture in the digital Age. Design and manufacturing. New York and London, Taylor \& Francis. 
Chien, S. F., \& Yeh, Y. T. (2012). On creativity and parametric design-A preliminary study of designer's behaviour when employing parametricdesign tools. In Proceedings of 30th International Conference on Education and Research in Computer Aided Architectural Design in Europe, Prague, Czech Republic (Vol. 1214).

Cropley, D. H., Kaufman, J. C., \& Cropley, A. J. (2011). Measuring creativity for innovation management. Journal of Technology Management \& Innovation, 6(3), 13-30.

Iordanova, I., Tidafi, T., Guité, M., De Paoli, G., \& Lachapelle, J. (2009). Parametric methods of exploration and creativity during architectural design. CAADFutures.

Iordanova, I., Tidafi, T., Guité, M., De Paoli, G., \& Lachapelle, J. (2009). Parametric methods of exploration and creativity during architectural design: A Case study in the design studio. Paper presented at the Proceedings of 13th International Conference on Computer Aided Architectural Design Futures, Montréal, Canada.

Lee, J. H., Gu, N., Jupp, J., \& Sherratt, S. (2014). Evaluating creativity in parametric design processes and products: a pilot study. In Design Computing and Cognition'12 (pp. 165183). Springer, Dordrecht.

Lee, Y. (2015). The parametric design genealogy of Zaha Hadid. Journal of Asian Architecture and Building Engineering, 14(2), 403-410.

Ostwald, M. J. (2012). Systems and enablers: Modeling the impact of contemporary computational methods and technologies on the design process. In Computational Design Methods and Technologies: Applications in CAD, CAM and CAE Education (pp. 1-17). IGI Global.

Oxman, R. (2008). Digital architecture as a challenge for design pedagogy: Theory, knowledge, models and medium. Design Studies, 29(2), 99-120.

Qian, C. Z., Chen, V. Y., \& Woodbury, R. F. (2007, October). Participant observationcan discover design patterns in parametric modeling. In Proceedings of 27th International Conference on the Association for Computer Aided Design in Architecture, Halifax, UK (Vol. 17).

Schnabel, M. A. (2007). Parametric designing in architecture. In Computer-Aided Architectural Design Futures (CAADFutures) 2007 (pp. 237-250). Springer, Dordrecht.

Schumacher, P. (2009). Parametricism: A new global style for architecture and urban design.

Schumacher, P., Shuojiong, Z., \& Unstudio, L. D. (2012). Parametricism the parametric paradigm and the formation of a new style. Time Architecture, 5, 007.

Yu, R., Gu, N., \& Ostwald, M. (2013). Comparing designers' problem-solving behavior in a parametric design environment and a geometric modeling environment. Buildings, 3(3), 621-638.

Zarei, Y. (2012). The challenges of paramteric design in architecture today: Mapping the design practice (Doctoral dissertation, Thesis-MPhil). 